\title{
Mean Weight Behavior of Coupled LMS Adaptive Systems Applied to Acoustic Feedback Cancellation in Hearing Aids
}

\author{
Yasmín Montenegro M. ${ }^{1}$ and José C. M. Bermudez ${ }^{2}$ \\ ${ }^{1}$ University of Antofagasta - Chile \\ ${ }^{2}$ Federal University of Santa Catarina - Brazil \\ ymontenegrom@yahoo.com.mx, j.bermudez@ieee.org*
}

\begin{abstract}
This paper presents a transient analysis of a hearing aids adaptive feedback canceller. The system employs an LMS adaptive estimator and an LMS adaptive predictor operating simultaneously. The nature of the practical problem makes the input to the adaptive estimator and the interference to its output statistically correlated. First, a modification is proposed to the original structure that accelerates convergence without compromising the cancellation level. The modified structure is then analyzed for slow adaptation and for an autoregressive input signal. Analytical models are derived for the mean behavior of the adaptive weights. Monte Carlo simulations verify the accuracy of the derived model.
\end{abstract}

Keywords: Adaptive systems, feedback, LMS, hearing aids.

\section{Introduction}

About $10 \%$ of the world's population suffers from hearing loss [1] that represents a serious limitation. The most common remedy for the hearing-impaired is a hearing aid [1]. However, user benefits are still limited due to the lack of effective algorithms that exploit all the digital signal processing potential. The occlusion effect and the acoustic feedback are among the main user complaints. Acoustic feedback is perceived as a distortion of the desired signal, limiting the maximum hearing aid's stable gain. Fig. 1] shows the basic structure of an adaptive feedback canceller. The acoustic feedback path is modelled by $\boldsymbol{w}^{0}$. The adaptive filter $\boldsymbol{w}(n)$ produces an estimate $\hat{y}(n)$ of the feedback signal $y(n)$. This estimate is subtracted from the microphone signal. $g(n)$ provides amplification. Ideally, $\hat{y}(n)$ converges to $y(n)$ for $n$ large. Then, only the input signal $x(n)$ is preserved at the hearing aids input $e(n)$ so that $u(n)=g(n) x(n)$.

Several strategies have been proposed to control acoustic feedback in hearing aids 2, 3, 4, 5, 6]. Continuously adaptive feedback cancellation is the most promising strategy because feedback depends strongly on the acoustical environment.

\footnotetext{
* This work has been partially supported CNPq under grants Nos. 307024/2006-7 and 470792/2006-0, by CAPES and by the University of Antofagasta-Chile.
} 


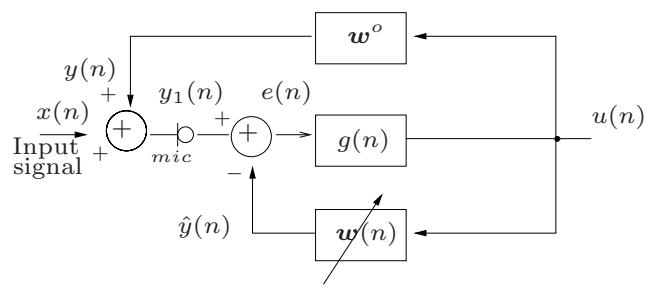

Fig. 1. Basic adaptive feedback cancellation in hearing aids

However, mean-square estimation of $y(n)$ in Fig. 1 leads to a biased solution because $x(n)$ and $u(n)$ are correlated. Several solutions proposed to reduce this bias introduce decorrelating operations such as delays or non-linearities in the forward path $g(n)$ or in the adaptive filter path [3, 4]. The use of restrictions in the adaptive canceller has also been proposed [5, 6]. Unfortunately, none of these solutions completely cancels the acoustic feedback.

Alternative solutions recently proposed rely on the direct method of closedloop identification [7. In this method, the data used for identification are obtained in closed loop but the identification is performed using an open loop model. Recent approaches assume a model for the input signal and apply the prediction-error method (PEM) to reduce the steady-state bias [8, 9]. The performance of these systems has been studied only in steady-state. Though steadystate behavior is an important performance measure for adaptive systems, it does not provide sufficient information on which to base the system design. Transient algorithm behavior is crucial for real-time applications such as hearing aids. Most of the recent structures incorporate more than one adaptive filter operating simultaneously, and little knowledge is available about the transient behavior of such adaptive structures.

This paper studies the transient behavior of the adaptive hearing aid feedback cancellation system recently proposed in [9]. The adaptive filter (called shadow filter) works offline while a fixed cancellation filter operates in the actual signal path. The adaptive filter weights are periodically used to update the cancellation filter response. The adaptive filter updating structure includes an adaptive prediction error filter $(\mathrm{PEF})$ to reduce the solution bias. Both adaptive filters are adapted simultaneously. A similar adaptive system has been analyzed in [10]. However, the analysis in [10 did not consider any additive interference to the unknown system output. In hearing aids, such interference is the desired signal $x(n)$, which is correlated to the adaptive filter input through the hearing aid amplifier. This feedback loop structure leads to a non-trivial transient analysis problem.

It is initially argued that the adaptive prediction error filter (PEF) should be applied to the output signal, instead of the estimation error as proposed in 9. This change results in faster convergence without affecting steady-state performance. Then, an analytical model is derived for the mean behavior of the adaptive weights of the modified structure for autoregressive input signals and 


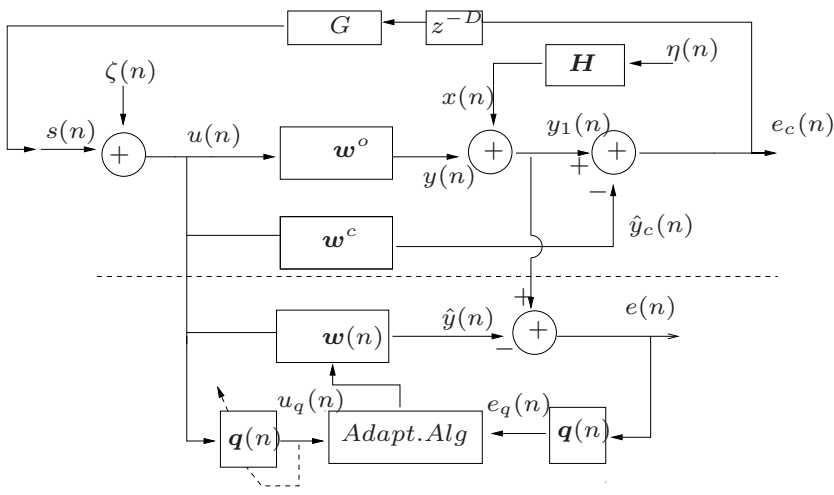

Fig. 2. Structure for feedback cancellation in hearing aids. Modified from 9 ]

under the assumption of slow adaptation. Monte Carlo simulation results show excellent agreement with the theoretical model.

\section{Adaptive System Studied}

Fig. 2 shows the adaptive feedback cancellation system studied. $x(n)$ is the desired signal. System $\boldsymbol{H}$ and a white Gaussian noise $\eta(n)$ define a parametric model for $x(n) . \zeta(n)$ is a zero-mean white noise required for identifiability of the feedback path when $x(n)$ includes periodic components [9]. $\boldsymbol{w}^{o}$ is the feedback path to be identified. $\boldsymbol{w}(n)$ is the adaptive estimation filter. $e_{c}(n)$ and $u(n)$ are, respectively, the input and output signals of the hearing aid, which is modeled by a gain $G$ and a delay $D \cdot \boldsymbol{w}^{c}$ is the feedback estimation filter implemented in the signal path. $\boldsymbol{w}^{c}$ is periodically updated with the coefficients of $\boldsymbol{w}(n)$ following some updating policy 9. The adaptive prediction error filter $\boldsymbol{q}(n)$ whitens $u(n)$ before its use by the adaptive algorithm. The same filter $\boldsymbol{q}(n)$ is also applied to $e(n)$. Note that the top part (above broken line) of Fig. 2 operates as an stationary system during adaptation of the bottom part. Therefore, signals $u(n)$ and $y_{1}(n)$ are considered stationary during adaptation of $\boldsymbol{w}(n)$ and $\boldsymbol{q}(n)$.

The structure in Fig. 2 is the same proposed in [9] except for the adaptive predictor location. In [9] the adaptive predictor was applied to the error signal $e(n)$ and copied to filter $u(n)$. Here we propose the structure shown in Fig. 2, This solution pre-whitens the adaptive algorithm input signal and speeds-up convergence of stochastic gradient algorithms [10] whithout affecting steadystate behavior of the system.

Under certain conditions, the structure in 9] can provide an unbiased feedback path identification. If $x(n)$ is an autoregressive process, it is shown in [9] that $e(n)$ converges to $x(n)$ and the adaptive PEF (applied to $e(n))$ converges to $\boldsymbol{H}^{-1}$. From Fig. 2] and using the subscript $q$ to refer to a signal filtered by $\boldsymbol{q}(n)$,

$$
e_{q}(n)=y_{1_{q}}(n)-\hat{y}_{q}(n) .
$$


Writing the PEF coefficient vector as $\boldsymbol{q}(n)=\left[1 \boldsymbol{p}^{T}(n)\right]^{T}, \hat{y}_{q}(n)=\hat{y}(n)+$ $\sum_{i=1}^{M} p_{i}(n) \hat{y}(n-i)=\boldsymbol{u}_{q}^{T}(n) \boldsymbol{w}(n)$ and

$$
e_{q}(n)=y_{1_{q}}(n)-\boldsymbol{u}_{q}^{T}(n) \boldsymbol{w}(n) .
$$

It is easy to show that $\mathrm{E}\left[e_{q}^{2}(n)\right]$ is minimized for $\boldsymbol{w}(n)=\hat{\boldsymbol{w}}$ where $\hat{\boldsymbol{w}}=\boldsymbol{R}_{u_{q} u_{q}}^{-1} \boldsymbol{r}_{q}$ with $\boldsymbol{R}_{u_{q} u_{q}}=\mathrm{E}\left\{\boldsymbol{u}_{q}(n) \boldsymbol{u}_{q}^{T}(n)\right\}$ and $\boldsymbol{r}_{q}^{T}=\mathrm{E}\left\{y_{q 1}(n) \boldsymbol{u}_{q}^{T}(n)\right\}$. Using $y_{q 1}(n)=$ $x_{q}(n)+\boldsymbol{u}_{q}^{T}(n) \boldsymbol{w}^{o}$ we have,

$$
\hat{\boldsymbol{w}}=\boldsymbol{R}_{u_{q} u_{q}}^{-1} \mathrm{E}\left\{x_{q}(n) \boldsymbol{u}_{q}(n)\right\}+\boldsymbol{w}^{o}
$$

which shows that the solution bias is controlled by $\mathrm{E}\left\{x_{q}(n) \boldsymbol{u}_{q}(n)\right\}$.

In 9] $\boldsymbol{q}(n)$ is adapted to whiten $e(n)$ and converges to the PEF for $x(n)$ upon convergence of $\boldsymbol{w}(n)$. Thus, $x_{q}(n)$ becomes uncorrelated to $u_{q}(n)$ in steady-state, yielding $\mathrm{E}\left\{x_{q}(n) \boldsymbol{u}_{q}(n)\right\}=0$ for $\mathrm{E}\left\{x_{q}(n)\right\}=0$, and the solution (3) becomes unbiased. It can be shown that the PEF applied to $u(n)$ as shown in Fig. 2 also converges to $\boldsymbol{H}^{-1}$. Thus, employing the adaptive PEF to either $e(n)$ or $u(n)$ leads to an unbiased steady-state solution. In steady-state both $e(n)$ and $u(n)$ are whitened by $\boldsymbol{q}(n)$. However, this is not true during transient because $\boldsymbol{q}(n)$ is the optimum PEF for $e(n)$ only upon convergence of $\boldsymbol{w}(n)$. On the other hand, the adaptive $\boldsymbol{q}(n)$ in Fig. 2 whitens $u(n)$ independently of $\boldsymbol{w}(n)$. Thus, the benefits of the PEF are available also during transient phase and improve overall convergence.

Fig. 3 shows Monte Carlo (MC) simulation results for the mean-square deviation (MSD) $\mathrm{E}\left\{\left\|\boldsymbol{w}(n)-\boldsymbol{w}^{o}\right\|^{2}\right\}$ for both structures using the LMS algorithm for $\boldsymbol{w}^{o}=[-0.0016,0.0016,0.0046,0.0502,-0.0691]^{T}$ and $H(z)=1 /\left(1-1.5 z^{-1}+\right.$ $z^{-2}-0.25 z^{-3}$ ] [1]. Step sizes equal to $10^{-4}$ were used for both the LMS estimator and the LMS PEF. Also $\sigma_{\eta}^{2}=0.1875$ and $\sigma_{\zeta}^{2}=10^{-5}$. The faster convergence of the structure of Fig. 2 is clear.

\section{Analysis}

The following analysis considers the stationarity of the top part of the system in Fig. 2 and assumes the input signals stationary for the adaptation period.

\subsection{Mean Weight Behavior for the Estimator}

Consider orders $N$ and $M$ for the estimator $\boldsymbol{w}(n)$ and for the PEF $\boldsymbol{q}(n)$, respectively. Defining the $N \times 1$ weight error vector $\boldsymbol{v}(n)=\boldsymbol{w}(n)-\boldsymbol{w}^{o}$, the LMS update for the estimator in Fig. 2 is

$$
\boldsymbol{v}(n+1)=\boldsymbol{v}(n)+\mu e_{q}(n) \boldsymbol{u}_{q}(n)
$$

where $e_{q}(n)=\boldsymbol{q}^{T}(n) \boldsymbol{e}(n)$ with $\boldsymbol{e}(n)=[e(n), \ldots, e(n-N+1)]^{T}$ and $\mu$ is the adaptation step. 


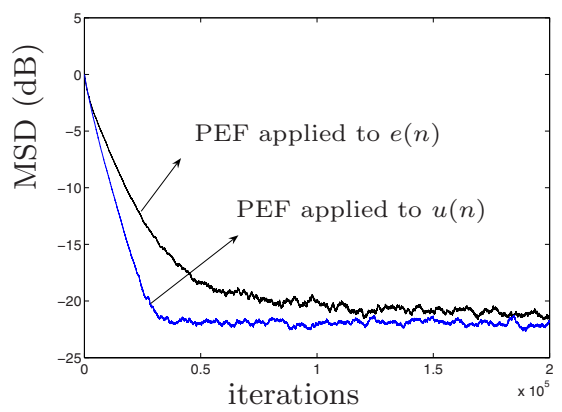

Fig. 3. Estimator MSD using the adaptive PEF on $e(n)$ and on $u(n)$

Assuming slow adaptation of both $\boldsymbol{q}(n)$ and $\boldsymbol{w}(n)$, we use the approximation $\boldsymbol{w}(n) \approx \ldots \approx \boldsymbol{w}(n-N+1)[10$. Under this approximation, $\boldsymbol{e}(n)=\boldsymbol{x}(n)-$ $\boldsymbol{U}(n) \boldsymbol{v}(n)$ where $\boldsymbol{U}(n)$ is an $M \times N$ input vector matrix with columns $\boldsymbol{u}(n), \cdots$, $\boldsymbol{u}(n-N+1)$. Thus, $e_{q}(n)$ can be written as

$$
e_{q}(n)=x_{q}(n)-\boldsymbol{u}_{q}^{T}(n) \boldsymbol{v}(n) .
$$

Using (5) in (4), taking the expectation and neglecting the correlation between $\boldsymbol{u}_{q} \boldsymbol{u}_{q}^{T}(n)$ and $\boldsymbol{v}(n)[12$ yields

$$
\begin{aligned}
\mathrm{E}\{\boldsymbol{v}(n+1)\}= & \mathrm{E}\{\boldsymbol{v}(n)\}-\mu \overbrace{\mathrm{E}\left\{\boldsymbol{u}_{q}(n) \boldsymbol{u}_{q}^{T}(n)\right\} \mathrm{E}\{\boldsymbol{v}(n)\}}^{\boldsymbol{\alpha}} \\
& +\underbrace{\mu \underbrace{}_{\mathrm{E}\left\{x_{q}(n) \boldsymbol{u}_{q}(n)\right\}}}_{\boldsymbol{\beta}}
\end{aligned}
$$

where $\boldsymbol{u}_{q}(n)=u(n)+\sum_{i=1}^{M} p_{i}(n) \boldsymbol{u}(n-i)$ and $x_{q}(n)=x(n)+\sum_{j=1}^{M} p_{j}(n) x(n-j)$ with $\boldsymbol{u}(n-i)=[u(n-i), \ldots, u(n-i-M)]^{T}$. Neglecting the fluctuations of $\boldsymbol{p}(n)$ and the correlations between $\boldsymbol{p}(n)$ and both $u(n)$ and $x(n)$ (slow adaptation), the $i$ th components of $\boldsymbol{\alpha}$ and $\boldsymbol{\beta}, i=0, \ldots, N-1$, in (6) are given by

$$
\begin{gathered}
\alpha_{i}(n)=\sum_{j=0}^{N-1} \sum_{k=0}^{M} \sum_{l=0}^{M} \mathrm{E}\left\{q_{k}(n)\right\} \mathrm{E}\left\{q_{l}(n)\right\} r_{u}(l+j-i-k) \mathrm{E}\left\{v_{j}(n)\right\} \\
\beta_{i}(n)=\sum_{j=0}^{M} \sum_{k=0}^{M} \mathrm{E}\left\{q_{j}(n)\right\} \mathrm{E}\left\{q_{k}(n)\right\} r_{x u}(k+i-j)
\end{gathered}
$$

where $r_{u}(l)=\mathrm{E}[u(n) u(n-l)], r_{x u}(l)=\mathrm{E}[x(n) u(n-l)], q_{0}(n)=1$ and $q_{\ell}(n)=$ $p_{\ell}(n)$ for $\ell=1, \ldots, M$. This completes the model for $\mathrm{E}\{\boldsymbol{v}(n)\}$.

\subsection{Mean Weight Behavior for the Predictor}

The predictor LMS weight update equation is $\boldsymbol{p}(n+1)=\boldsymbol{p}(n)-\rho u_{q}(n) \boldsymbol{u}(n-1)$ with $\rho$ as adaptation step. 
Taking the expectation under the assumption of slow adaptation yields

$$
\mathrm{E}[\boldsymbol{p}(n+1)]=\left[I-\rho \boldsymbol{R}_{u u}(0)\right] \mathrm{E}[\boldsymbol{p}(n)]-\rho \boldsymbol{r}_{u}(1)
$$

for $\boldsymbol{R}_{u u}(0)=\mathrm{E}\left[\boldsymbol{u}(n-1) \boldsymbol{u}^{T}(n-1)\right]$ and $\boldsymbol{r}_{u}(1)=\mathrm{E}[u(n) \boldsymbol{u}(n-1)]$.

\subsection{Correlations}

Evaluation of $r_{u}(l)$ and $r_{x u}(l)$ is necessary to complete the analysis above. These correlations are obtained from the analysis of the stationary closed loop subsystem in the top part of Fig. 2 for an autoregressive input $x(n)$.

Autocorrelation $\boldsymbol{r}_{\boldsymbol{u}}(\boldsymbol{l})$. Defining the time-invariant weight error vector $\boldsymbol{v}^{c}=$ $\boldsymbol{w}^{c}-\boldsymbol{w}^{o}$, we have

$$
u(n)=\gamma(n)-G \boldsymbol{u}^{T}(n-D) \boldsymbol{v}^{c}
$$

where $\gamma(n)=\zeta(n)+G x(n-D)$. Taking the $\mathrm{z}$-transform of (10) yields

$$
H_{u}(z)=\frac{U(z)}{\Gamma(z)}=\frac{1}{1+G \boldsymbol{\psi}\left(z^{-1}\right) \boldsymbol{v}^{c} z^{-D}}
$$

with $\psi\left(z^{-1}\right)=\left[1, z^{-1}, z^{-2}, \cdots, z^{-N+1}\right]^{T}$. Since $\zeta(n)$ is independent of $x(n)$,

$$
\begin{gathered}
R_{u}(z)=\frac{R_{\gamma u}(z)}{1+G \boldsymbol{\psi}\left(z^{-1}\right) \boldsymbol{v}^{c} z^{-D}} \\
R_{\gamma u}(z)=\frac{R_{\zeta}(z)}{1+G \boldsymbol{\psi}(z) \boldsymbol{v}^{c} z^{D}}+\frac{G^{2} R_{x}(z)}{1+G \boldsymbol{\psi}(z) \boldsymbol{v}^{c} z^{D}}
\end{gathered}
$$

where $R_{u}(z)$ is the complex spectral density of $u(n)$ and $R_{\gamma u}(z)$ is the complex cross-spectral density of $u(n)$ and $\gamma(n)$.

For $x(n)$ autoregressive, $R_{x}(z)=H(z) H^{*}\left(1 / z^{*}\right) \sigma_{\eta}^{2}$ with

$$
H(z)=\frac{1}{\prod_{i=1}^{M}\left(1+a_{i} z^{-1}\right)} .
$$

Assuming stability of (11) and using the Residue Theorem on (13), straightforward calculation leads to

$$
\begin{aligned}
r_{\gamma u}(l)= & \sigma_{\zeta}^{2} \delta(l)+\sum_{k=1}^{M} \frac{G^{2} \sigma_{\eta}^{2}\left(-a_{k}\right)^{M}\left(-a_{k}\right)^{l-1}}{\prod_{\substack{i=1 \\
k \neq i}}^{M}\left(a_{i}-a_{k}\right) \prod_{j=1}^{M}\left(1-a_{j} a_{k}\right)} \\
& \times \frac{1}{\left(1+G \boldsymbol{\psi}\left(-a_{k}\right) \boldsymbol{v}^{c}\left(-a_{k}\right)^{D}\right)}, \quad l \geq 0 .
\end{aligned}
$$

Finally, it follows from (12) that

$$
r_{u}(l)=r_{\gamma u}(l)-G \boldsymbol{r}_{u}^{T}(l-D) \boldsymbol{v}^{c}
$$

where $\boldsymbol{r}_{u}(l-D)=\left[r_{u}(l-D), \ldots, r_{u}(l-D-N+1)\right]^{T}$. 
Cross-correlation $\boldsymbol{r}_{\boldsymbol{x} \boldsymbol{u}}(\boldsymbol{l})$. We have that $r_{x u}(l)=\mathrm{E}\{x(n+l) u(n)\}=G h(-l) *$ $r_{x}(l+D)$ where $h(n)$ is the inverse z-transform of (14) and $*$ means convolution. Thus,

$$
R_{x u}(z)=G H^{*}\left(1 / z^{*}\right) R_{x}(z) z^{D} .
$$

Substituting (14) and $R_{x}(z)=H(z) H^{*}\left(1 / z^{*}\right) \sigma_{\eta}^{2}$ in (17) and using the Residue Theorem yields

$$
\begin{aligned}
r_{x u}(l)= & \sum_{k=1}^{M} \frac{G \sigma_{\eta}^{2}\left(-a_{k}\right)^{D+M}\left(-a_{k}\right)^{l-1}}{\prod_{\substack{i=1 \\
k \neq i}}^{M}\left(a_{i}-a_{k}\right) \prod_{j=1}^{M}\left(1-a_{j} a_{k}\right)} \\
& \times \frac{1}{\left(1+G \boldsymbol{\psi}\left(-a_{k}\right) \boldsymbol{v}^{c}\left(-a_{k}\right)^{D}\right)}, \quad l \geq 0
\end{aligned}
$$

and

$$
\begin{aligned}
r_{x u}(l)= & \sum_{k=1}^{M} \frac{G \sigma_{\eta}^{2}\left(-a_{k}\right)^{D+M}}{\left(-a_{k}\right)^{l+1} \prod_{\substack{i=1 \\
k \neq i}}^{M}\left(a_{i}-a_{k}\right) \prod_{j=1}^{M}\left(1-a_{j} a_{k}\right)} \\
& \times \frac{1}{\left(1+G \boldsymbol{\psi}\left(-a_{k}\right) \boldsymbol{v}^{c}\left(-a_{k}\right)^{D}\right)}, \quad l<0 .
\end{aligned}
$$

\section{Simulation Results}

This section presents Monte Carlo (MC) simulations to verify the theoretical models. The parameters used in the example were $\mu=0.001, \rho=0.001$, $\sigma_{x}^{2}=1$ (using $\left.\sigma_{\eta}^{2}=0.1875\right), \sigma_{\zeta}^{2}=10^{-5}$ and $H(z)=1 /\left(1-1.5 z^{-1}+z^{-2}-\right.$ $0.25 z^{-3}$ ) (thus the optimal predictor is $\boldsymbol{p}^{o}=[-1.5 ; 1 ;-0.25]^{T}$ ). Two feedback paths are utilized: $\boldsymbol{w}_{1}^{o}=[-0.0016,0.0016,0.0046,0.0502,-0.0691]^{T}$ and $\boldsymbol{w}_{2}^{o}=$ $[-0.0306,0.0101,0.0406,0.0650,0.0250]^{T}$ (first 5 samples of an actual feedback

(a)

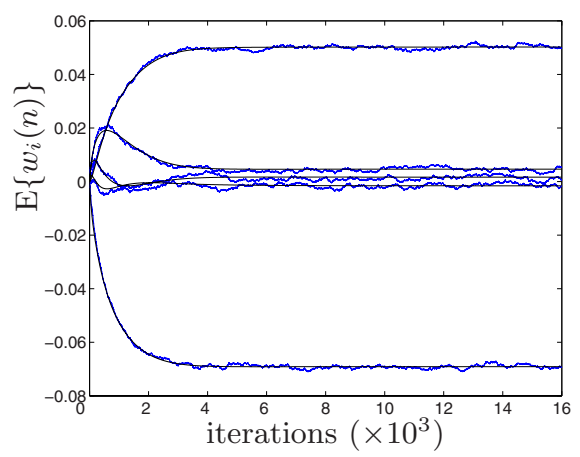

(b)

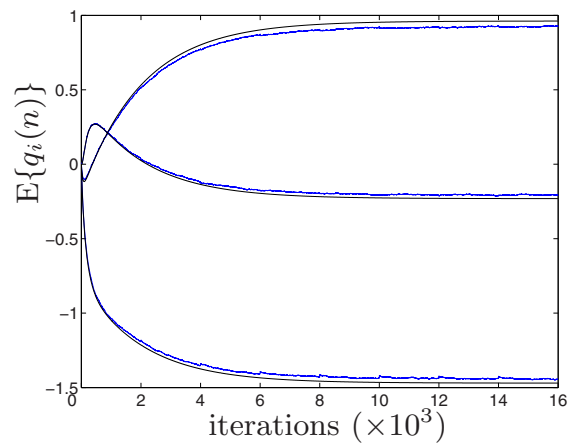

Fig. 4. Coefficient misadjustment in the filters: (a) Estimator and (b) Predictor for the feedback path $\boldsymbol{w}_{1}^{o}$ 
(a)

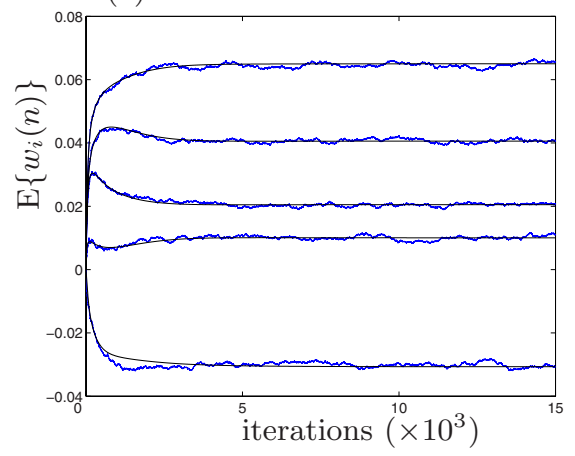

(b)

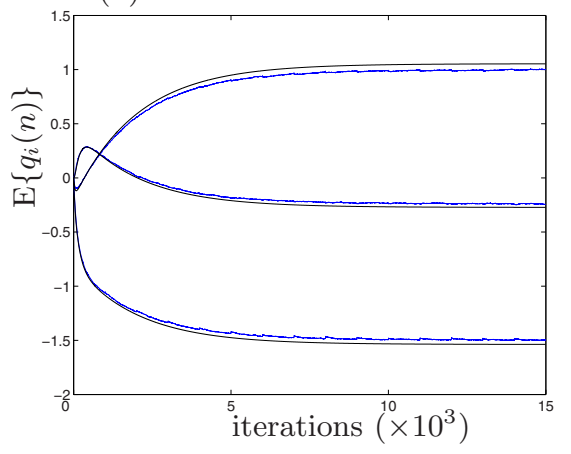

Fig. 5. Coefficient misadjustment in the filters: (a) Estimator and (b) Predictor for the feedback path $\boldsymbol{w}_{2}^{o}$

path response). The Figs. 4 and 5 show the theoretical mean coefficient behaviors (smooth curves) and the MC simulations (ragged curves) averaged over 150 runs for the estimator and for the predictor. These results show excellent agreement between simulation results and the theoretical model provided by (6), (9), (16), (18) and (19) both in the transient and in the steady-state phases of adaptation.

\section{Conclusions}

This paper has presented a transient analysis of two coupled adaptive systems, one LMS estimator and one LMS predictor, when applied to feedback cancellation in hearing aids. A modification was proposed to the structure of [9]. The modification increases the convergence speed of the estimator filter without compromising the steady-state performance. Analytical models for first moment of both the adaptive filter weight vectors were derived. Monte Carlo simulation results illustrate the accuracy of the new models both during transient and in steady-state.

\section{References}

1. Chung, K.: Challenges and recent develpoments in heraing aids part.1 speech undestanding in noise, microphone technologies and noise reduction algorithms. Trens In Amplification 8(3), 83-124 (2004)

2. Kates, J.M.: Feedback cancellation in hearing aids: results from a computer simulation. IEEE Trans. on Speech and audio Processing 39(3), 553-562 (1991)

3. Joson, H.A.L., Asano, F., Toshio, Y.S.S.: Adaptive feedback cancellation with frequency compresion for hearing aids. J. Acoust. Society of America 94(6), 3248-3254 (1993)

4. Siqueira, M., Alwan, A.: Steady-state analysis of continuous adaptation in acoustic feedback reduction systems for hearing-aids. IEEE Trans. on speech and audio processing 8(4), 443-453 (2000) 
5. Kates, J.M.: Constrained adaptation for feedback cancellation in hearing aids. J. Acoust. Society of America 106(2), 1010-1019 (1999)

6. Benesty, J., Huang, Y.: Adaptive Signal Processing: Applications to Real-World Problems. Springer, New York (2003)

7. Ljung, L.: System identification, theory for the user. Prentice Hall, Englewood Cliffs (1987)

8. Hellgren, J., Forssell, U.: Bias of feedback cancellation algorithms in hearing aids based on direct closed loop identification. IEEE Trans. Speech and audio processing 9(7), 906-913 (2001)

9. Spriet, A.I., Proudler, M., Wouters, J.: Adaptive feedback cancellation in hearing aids with linear prediction of the desired signal. IEEE Trans. on Signal processing 53(10), 3749-3763 (2005)

10. Mboup, M.B., Bershad, N.: Lms coupled adaptive prediction and system identification: a statistical model and transient mean analysis. IEEE Transaccion Signal Processing 42(10), 2607-2615 (1994)

11. Ho, K.C., Blunt, S.D.: Rapid identification of a sparse impulse response using an adaptive algorithm in the haar domain. IEEE Trans. on Signal Processing 51(3), 628-638 (2003)

12. Minkoff, J.: Comment: On the unnecessary assumption of statistical independence between reference signal and filter weights in feedforward adaptive systems. IEEE Trans. on Signal Processing 49(5), 1109 (2001) 